\title{
Some Remarks on the Sumudu and Laplace Transforms and Applications to Differential Equations
}

\author{
Adem Kılıçman $^{1}$ and Hassan Eltayeb ${ }^{2}$ \\ ${ }^{1}$ Institute of Mathematical Research (INSPEM) and Department of Mathematics, \\ Universiti Putra Malaysia, Selangor, 43400 Serdang, Malaysia \\ ${ }^{2}$ Mathematics Department, College of Science, King Saud University, P.O. Box 2455, \\ Riyadh 11451, Saudi Arabia \\ Correspondence should be addressed to Adem K1lıçman, akilicman@putra.upm.edu.my \\ and Hassan Eltayeb, hgadain@ksu.edu.sa
}

Received 10 October 2011; Accepted 20 November 2011

Academic Editor: K. Djidjeli

Copyright (c) 2012 A. K1lıçman and H. Eltayeb. This is an open access article distributed under the Creative Commons Attribution License, which permits unrestricted use, distribution, and reproduction in any medium, provided the original work is properly cited.

We study the relationship between Sumudu and Laplace transforms and further make some comparison on the solutions. We provide some counterexamples where if the solution of differential equations exists by Laplace transform, the solution does not necessarily exist by using the Sumudu transform; however, the examples indicate that if the solution of differential equation by Sumudu transform exists then the solution necessarily exists by Laplace transform.

\section{Introduction}

In order to solve the differential equations, the integral transform is extensively applied and thus there are several works on the theory and application of integral transforms. In the sequence of these transforms, Watugala introduced a new integral transform, named the Sumudu transform, and further applied it to the solution of ordinary differential equation in control engineering problems; see [1]. For further details and properties of Sumudu transform see [2-7] and many others. The Sumudu transform is defined over the set of the functions

$$
A=\left\{f(t): \exists M, \tau_{1}, \tau_{2}>0,|f(t)|<M e^{t / \tau_{j}}, \text { if } t \in(-1)^{j} \times[0, \infty)\right\}
$$


by the following formula:

$$
\bar{f}(u)=S[f(t) ; u]=: \int_{0}^{\infty} f(u t) e^{-t} d t, \quad u \in\left(-\tau_{1}, \tau_{2}\right) .
$$

The existence and the uniqueness was discussed in [8]; for further properties of Sumudu transform and its derivatives, we refer to [2]. In [3], some fundamental further properties of Sumudu transform were also established.

Similarly, this new transform was applied to the one-dimensional neutron transport equation in [9]. In fact, one can easily show that there is strong relationship between Sumudu and other integral transforms. In particular, the relation between Sumudu transform and Laplace transforms was proved in [8].

Further in [6], the Sumudu transform was extended to the distributions (generalized functions) and some of their properties were also studied in [10]. Recently, Kiliçman et al. applied this transform to solve the system of differential equations; see [7, 11].

Now, let us recall the following definition which is held in Estrin and Higgins; see [12]; the double Laplace Transform is defined by

$$
L_{x} L_{t}[f(x, s)]=F(p, s)=\int_{0}^{\infty} e^{-p x} \int_{0}^{\infty} e^{-s t} f(x, t) d t d x,
$$

where $x, t>0$ and $p, s$ are complex numbers. The double Sumudu transform of second partial derivative with respect to $x$ is given by

$$
\begin{aligned}
S_{2}\left[\frac{\partial^{2} f(t, x)}{\partial x^{2}} ;(v, u)\right] & =\frac{1}{u v} \int_{0}^{\infty} \int_{0}^{\infty} e^{-(t / v+x / u)} \frac{\partial^{2} f(t, x)}{\partial x^{2}} d t d x \\
& =\frac{1}{u} \int_{0}^{\infty} e^{-t / v}\left(\frac{1}{v} \int_{0}^{\infty} e^{-x / u} \frac{\partial^{2} f(t, x)}{\partial x^{2}} d x\right) d t .
\end{aligned}
$$

Then, the interior integral is given by

$$
\frac{1}{u} \int_{0}^{\infty} e^{-x / v} \frac{\partial^{2} f(t, x)}{\partial x^{2}} d x=\frac{1}{u^{2}} F(t, v)-\frac{1}{u^{2}} F(t, 0)-\frac{1}{u} \frac{\partial f(t, 0)}{\partial x}
$$

By taking Sumudu transform with respect to $t$ for (1.5), we get double Sumudu transform in the form of

$$
S_{2}\left[\frac{\partial^{2} f(t, x)}{\partial x^{2}} ;(u, v)\right]=\frac{1}{u^{2}} F(v, u)-\frac{1}{u^{2}} F(0, u)-\frac{1}{u} \frac{\partial f(0, u)}{\partial x} .
$$

Similarly, double Sumudu transform of $\partial^{2} f(t, x) / \partial t^{2}$ is given by

$$
S\left[\frac{\partial^{2} f(t, x)}{\partial t^{2}} ;(u, v)\right]=\frac{1}{v^{2}} F(v, u)-\frac{1}{v^{2}} F(v, 0)-\frac{1}{v} \frac{\partial f(v, 0)}{\partial t} .
$$


And double Laplace transform defined the first-order partial derivative as

$$
L_{x} L_{t}\left[\frac{\partial f(x, t)}{\partial x}\right]=p F(p, s)-F(0, s)
$$

by

The double Laplace transform for second partial derivative with respect to $x$ is given

$$
L_{x x}\left[\frac{\partial^{2} f(x, t)}{\partial^{2} x}\right]=p^{2} F(p, s)-p F(0, s)-\frac{\partial F(0, s)}{\partial x},
$$

and double Laplace transform for second partial derivative with respect to $t$ similarly as above is given by

$$
L_{t t}\left[\frac{\partial^{2} f(x, t)}{\partial^{2} t}\right]=s^{2} F(p, s)-s F(p, 0)-\frac{\partial F(p, 0)}{\partial t} .
$$

In a similar manner, the double Laplace transform of a mixed partial derivative can be deduced from single Laplace transform as

$$
L_{x} L_{t}\left[\frac{\partial^{2} f(x, t)}{\partial x \partial t}\right]=p s F(p, s)-p F(p, 0)-s F(0, s)-F(0,0)
$$

Our purpose here is to show the difference between Laplace transform and Sumudu transform by solving partial differential equations. In fact, the double Sumudu transform and double Laplace transform have a strong relationship that may be expressed either as

$$
\begin{aligned}
& \text { (I) } u v F(u, v)=\perp_{2}\left(f(x, y) ;\left(\frac{1}{u}, \frac{1}{v}\right)\right) \\
& \text { (II) } p s F(p, s)=\perp_{2}\left(f(x, y) ;\left(\frac{1}{p}, \frac{1}{s}\right)\right)
\end{aligned}
$$

where $\mathcal{L}_{2}$ represents the operation of double Laplace transform. In particular, this relation is best illustrated by the fact that the double Sumudu and double Laplace transforms interchange the image of $\sin (x+t)$ and $\cos (x+t)$. It turns out that

$$
\begin{aligned}
& S_{2}[\sin (x+t)]=\mathcal{L}_{2}[\cos (x+t)]=\frac{u+v}{(1+u)^{2}(1+v)^{2}}, \\
& S_{2}[\cos (x+t)]=\mathcal{L}_{2}[\sin (x+t)]=\frac{1}{(1+u)^{2}(1+v)^{2}} .
\end{aligned}
$$


Further, the double Laplace and Sumudu transforms interchange the images of the Dirac function, $\delta(x, t)=\delta(x) \delta(t)$ and the Heaviside function, $H(x, t)=H(x) \otimes H(t)$, since

$$
\begin{aligned}
& S_{2}[H(x, t)]=\mathcal{L}_{2}[\delta(x, t)]=1, \\
& S_{2}[\delta(x, t)]=\mathcal{L}_{2}[H(x, t)]=\frac{1}{u v},
\end{aligned}
$$

where the symbol $\otimes$ means the tensor product thus the relation between the double Sumudu and double Laplace transform of convolution was given by

$$
S_{2}[(f * * g)(t, x) ; v, u]=\frac{1}{u v} \rho_{2}[(f * * g)(t, x)]
$$

(see [10]).

Note that since many practical engineering problems involve mechanical or electrical systems acted upon by discontinuous or impulsive forcing terms, then the Sumudu transform can be effectively used to solve ordinary differential equations as well as partial differential equations in engineering problems; see [13]. In this paper, we study the relationship between Sumudu and Laplace transforms and further make some comparison on the solutions. Provide a counterexample where if the solution of differential equation by Laplace transform exists then it does not necessarily exist by using the Sumudu transform, however, if the solution of differential equation by Sumudu transform exist, then solution necessarily exists by Laplace transform. First of all we need the following concept related to the Sumudu transform of derivatives.

Proposition 1.1 (Sumudu Transform of Derivative). Let $f$ be differentiable on $(0, \infty)$ and let $f(t)=0$ for $t<0$. Suppose that $f^{\prime} \in L_{\mathrm{loc}}$. Then, $f^{\prime} \in L_{\mathrm{loc}}, \operatorname{dom}(S f) \subset \operatorname{dom}\left(f^{\prime}\right)$, and

$$
S\left(f^{\prime}\right)=\frac{1}{u} S(f)-\frac{1}{u} f(0+) \quad \text { for } u \in \operatorname{dom}(S(f))
$$

More generally, if $f$ is differentiable on $(c, \infty), f(t)=0$ for $t<0$, and $f^{\prime} \in L_{\mathrm{loc}}$, then

$$
S\left(f^{\prime}\right)=\frac{1}{u} S(f)-\frac{1}{u} e^{-c / u} f(c+) \quad \text { for } u \in \operatorname{dom}(S(f)) .
$$

Proof. For the proof of this proposition, see [14].

In general case, if $f$ is a differentiable function on $(a, b)$ with $a<b$, and $f(t)=0$ for $t<a$ or $t>b$ and $f^{\prime} \in L_{\mathrm{loc}}$, then, for all $u$,

$$
S\left(f^{\prime}\right)=\frac{1}{u} S(f)-\frac{1}{u} e^{-a / u} f(a+)+\frac{1}{u} e^{-b / u} f(b-) .
$$


Proposition 1.1 can be extended to higher derivatives, before extension, we introduce the following notation as in [11]. Let $P(x)=\sum_{k=0}^{n} a_{k} x^{k}$ be a polynomial in $x$, where $n \geq 0$ and $a_{n} \neq 0$. We define $M_{P}(x)$ to be the $1 \times n$ matrix of polynomials given by the matrix product:

$$
M_{P}(x)=\left(\begin{array}{lllll}
1 & x & x^{2} & \cdots & x^{n-1}
\end{array}\right)\left(\begin{array}{cccccc}
a_{1} & a_{2} & \cdot & \cdot & \cdot & a_{n} \\
a_{2} & a_{3} & \cdot & \cdot & a_{n} & 0 \\
a_{3} & \cdot & \cdot & a_{n} & 0 & 0 \\
\cdot & \cdot & \cdot & \cdot & \cdot & \cdot \\
a_{n} & 0 & \cdot & \cdot & \cdot & 0
\end{array}\right)
$$

For each complex number $x$, the map $M_{P}(x)$ defines a linear mapping of $\mathbb{C}^{n}$ into $\mathbb{C}$ in obvious way. We will write vectors $y$ in $\mathbb{C}^{n}$ as row vectors or column vectors interchangeably, whichever, is convenient, although when $M_{P}(x) y$ is to be compute and the matrix representation by (1.19) of $M_{P}(x)$ is used, then of course $y$ must be written as a column vector:

$$
M_{P}(x) y=\sum_{i=1}^{n} x^{i-1} \sum_{k=0}^{n-i} a_{i+k} y_{k}
$$

for any $y=\left(y_{0}, y_{1}, \ldots, y_{n-1}\right) \in \mathbb{C}^{n}$. If $n=0$, then $M_{P}(x)$ defines a unique linear mapping of $\{0\}=\mathbb{C}^{0}$ into $\mathbb{C}$ (empty matrix). In general, if $n>0$ and $f$ is $n-1$ times differentiable on an interval $(a, b)$, with $a<b$, then we shall write

$$
\begin{aligned}
& \varphi(f ; a ; n)=\left(f(a+), f^{\prime}(a+), \ldots, f^{(n-1)}(a+)\right) \in \mathbb{C}^{n}, \\
& \phi(f ; b ; n)=\left(f(b-), f^{\prime}(b-), \ldots, f^{(n-1)}(b-)\right) \in \mathbb{C}^{n} .
\end{aligned}
$$

If $a=0$, we write $\varphi(f ; n)$ for $\varphi(f ; 0 ; n)$. If $n=0$, then we define

$$
\varphi(f ; a ; 0)=\phi(f ; a ; 0)=0 \in \mathbb{C}^{0} .
$$

Now, we need to consider the transform of higher derivatives as follows.

Proposition 1.2 (Sumudu transform of higher derivatives). Let $f$ be $n$ times differentiable on $(0, \infty)$, and let $f(t)=0$ for $t<0$. Suppose that $f^{(n)} \in L_{\mathrm{loc}}$. Then, $f^{(k)} \in L_{\mathrm{loc}}$ for $0 \leq k \leq n-1$, $\operatorname{dom}(S f) \subset \operatorname{dom}\left(S f^{(n)}\right)$ and, for any polynomial $P$ of degree $n$,

$$
S[P(\dot{D}) f](u)=P(u) S(f)(u)-M_{P}(u) \varphi(f ; n)
$$

for $u \in \operatorname{dom}(S f)$. In particular,

$$
\left(S f^{(n)}\right)(u)=\frac{1}{u^{n}}(S f)(u)-\left(\frac{1}{u^{n}}, \frac{1}{u^{n-1}}, \ldots, \frac{1}{u}\right) \varphi(f ; n)
$$


(with $\varphi(f ; n)$ here written as a column vector). For $n=2$, one has

$$
\left(S f^{\prime \prime}\right)(u)=\frac{1}{u^{2}}(S f)(u)-\frac{1}{u^{2}} f(0+)-\frac{1}{u} f^{\prime}(0+) .
$$

Proof. For the proof of this proposition, see [8].

In general, if $f$ is differentiable on $(a, b)$ with $a<b$, and $f(t)=0$ for $t<a$ or $t>b$ and $f^{(n)} \in L_{\text {loc }}$ then we have, for all $u$,

$$
S[P(\dot{D}) f](u)=P(u)(S f)(u)-M_{P}(u)\left[e^{-a / u} \varphi(f ; a ; n)-e^{-b / u} \phi(f ; b ; n)\right] .
$$

\section{Solution of Differential Equations by Convolution Methods}

In this section, we give the solution of the following equation:

$$
a_{n} y^{(n)}+a_{n-1} y^{(n-1)}+\cdots+a_{1} y^{\prime}+a_{0} y=f
$$

on $(0, \infty)$. We prove an existence and uniqueness and provide a formula for the solution.

If we define $f$ and $y$ to be zero on $(-\infty, 0)$, then (2.1) is equivalent to the equation

$$
P(\dot{D}) y=f
$$

where

$$
P(x)=\sum_{k=0}^{n} a_{k} x^{k}
$$

First of all, we establish first an important result for homogeneous equation.

Theorem 2.1 (Properties of solution of the homogeneous equation). Let $n \geq 0$ and let $a_{k}(0 \leq$ $k \leq n)$ be complex constant such that $a_{n} \neq 0$. Let $y$ be $n$ differentiable on $(0, \infty)$ and zero $(-\infty, 0)$ and satisfy

$$
P(\dot{D}) y=0
$$

then one has the following.

(1) $y$ is infinitely differentiable on $(0, \infty)$.

(2) For every integer $k \geq 0$, the limits $y^{k}(0+)$ exist.

(3) If $n>0, y$ then is given (except at 0) by the formula

$$
y=S^{-1}\left[\frac{M_{P}(u)}{P(u)} \varphi(y ; n)\right]=M_{P}(\dot{D}) V \varphi(y ; n),
$$


where

$$
V=S^{-1}\left[\frac{1}{P}\right]
$$

Proof. To avoid trivial statements, suppose that $n>0$. Let $0<a<b$. The function $z=y\left(H_{a}-\right.$ $\left.H_{b}\right)$ is $n$ times differentiable on $(a, b)$, and $z$, and $z^{n}$ are locally integrable and $\operatorname{dom}(S(z))=\mathbb{R}$. By $(1.26)$ and the relation $\varphi(z ; a ; n)=\varphi(y ; a ; n), \phi(z ; b ; n)=\varphi(y ; b ; n)$; we have, for all $u$

$$
S[P(\dot{D}) z](u)=P(u)(S z)(u)-M_{P}(u)\left[e^{-a / u} \varphi(y ; a ; n)-e^{-b / u} \varphi(y ; b ; n)\right] .
$$

Since $P(\dot{D}) z=0$, we obtain for large $u$

$$
(S z)(u)=\frac{M_{P}(u)}{P(u)}\left[e^{-a / u} \varphi(y ; a ; n)-e^{-b / u} \varphi(y ; b ; n)\right] .
$$

Now, $(M p(u) / P(u))[\varphi(y ; a ; n)]$ is a proper rational function of $u$; there is a function $g_{a}$, analytic on $\mathbb{R}$, such that, for $u$ sufficiently large,

$$
\left(S g_{a} H\right)(u)=\frac{M_{P}(u)}{P(u)} \varphi(y ; a ; n)
$$

With $g_{b}$ defined analogously, we deduce from (2.8) and the shift rule that

$$
y(t)[H(t-a)-H(t-b)]=g_{a}(t-a) H(t-a)-g_{b}(t-b) H(t-b)
$$

if $a<t<b$, this gives

$$
y(t)=g_{a}(t-a)
$$

thus $y$ is analytic on every open interval $(a, b)$, with $0<a<b$; hence, $y$ is analytic on $(0, \infty)$. However, on the interval $(1,2)$, we have $y(t)=g_{1}(t-1)$. We conclude that this formula must hold for all $a>0$, which allows us to write

$$
y(t)=g_{1}(t-1) H(t)
$$

for all $t \neq 0$; from this formula, (1.1) and (1.2) follow immediately. Now, we write, for $t \neq 0$,

$$
y(t)=g_{1}(t-1)[H(t)-H(t-1)]+g_{1}(t-1) H(t-1) .
$$


The first term on the right is clearly Sumudu transformable. Similarly, the second term is also transformable since it is merely the translated function $\left(g_{1} H\right)(t-1)$, thus $y$ is Sumudu transformable. To obtain the formula (2.5), we apply (1.23) and get

$$
S[y](u)=\frac{M_{P}(u)}{P(u)} \varphi(y ; n) .
$$

Since $M_{P}(u) \varphi(y ; n)$ is a polynomial of degree less than $n$, by using Sumudu inverse transform, we obtain

$$
y=S^{-1}\left[\frac{M_{P}(u)}{P(u)} \varphi(y ; n)\right]=M_{P}(\dot{D}) V \varphi(y ; n) .
$$

Now, we extend the above theorem to the nonhomogeneous equation as follows.

Proposition 2.2. Let $a_{k}$ be as in the above theorem. Let $f$ be continuous on $(0, \infty)$ and zero on $(-\infty, 0)$ and let $f$ be locally integrable. Let $y$ be $n$ times differentiable on $(0, \infty)$ and zero on $(-\infty, 0)$ and satisfy

$$
P(\dot{D}) y=f
$$

then one has the following.

(1) $y^{n}$ is continuous on $(0, \infty)$ and locally integrable on $\mathbb{R}$.

(2) For $0 \leq k \leq n-1$, the limits $y^{(k)}(0+)$ exist.

(3) If $n>0$, then $y$ is given by the formula

$$
y=V * f+M_{P}(\dot{D}) V \varphi(y ; n) .
$$

Proof. The result is trivial if $n=0$. Suppose that $n>0$. Let $z=V * f$. if $f$ is continuous on an open interval $I$, then $V * f$ is $n$ times differentiable on $I$. We have $P(\dot{D}) z=f$. Also, consider that $z^{(n)}$ is continuous for $0 \leq k \leq n-1$, and hence $z^{(n)}$ is locally integrable on $\mathbb{R}$ and continuous on $(0, \infty)$. Let $w=y-z$. Then, $P(\dot{D}) w=0$. By using the above theorem, $w^{(n)}$ is locally integrable and $w^{(k)}(0+)$ exist for all $k \geq 0$, and we have $w=M_{P}(\dot{D}) V \varphi(y ; n)$. If we now write $y=w+z$, then the properties (1) and (2) follow immediately. Equation (2.17) also follows because $\varphi(z ; n)=0$ by using the statement if $f(t)=0$ for $t<c$, then $(V * f)^{(k)}(c)=0$ for $0 \leq k \leq n-1$, where $V=S^{-1}[1 / P(u)]$, and hence $\varphi(w ; n)=\varphi(y ; n)$.

In the next theorem, we provide a complete solution of a non-homogeneous equation.

Theorem 2.3 (Existence and Uniqueness). Let $f$ be continuous function on $(0, \infty)$, zero on $(-\infty, 0)$, and locally integrable. Let $\gamma \in \mathbb{C}^{n}$. Then, there exists a unique (except on zero) function $y$ that is $n$ times differentiable on $(0, \infty)$ and zero on $(-\infty, 0)$ satisfying

$$
P(\dot{D}) y=f, \quad \gamma=\varphi(y ; n)
$$


If $n>0$, then $y$ is given (except on zero) by

$$
y=V * f+S^{-1}\left[\frac{M_{P}(u) \gamma}{P(u)}\right]=V * f+M_{P}(\dot{D}) V \gamma
$$

Proof. Uniqueness is obvious by (2.17), thus $y$ is given by (2.19). To establish existence, we consider $y$ by (2.19). Since

$$
P(\dot{D}) M_{P}(\dot{D}) V \gamma=M_{P}(\dot{D})[P(\dot{D}) V] \gamma=0
$$

by taking Sumudu transform $P(\dot{D}) V$ and using (1.23), we have

$$
S[P(\dot{D}) V]=P(u) S[V]-M_{P}(u) \varphi(V ; n)=1-M_{P}(u) \varphi(V ; n) .
$$

This is a polynomial in $u$ which by virtue of the statement (if $f$ be Sumudu transformable and satisfy $f(t)=0$ for $t<0$. Then, $\lim _{u \rightarrow \infty} S[f](u)=0$ ) must be identically zero. Thus, $P(\dot{D}) V=0$, then (2.20) is true. We have

$$
P(\dot{D}) y=P(\dot{D})(V * f)=f
$$

To verify that $y$ satisfies the initial conditions, we first observe that by (2.17) we must have $y=V * f+M_{P}(\dot{D}) V \varphi(y ; n)$. We deduce that

$$
M_{P}(\dot{D}) V[r-\varphi(y ; n)]=0
$$

Taking Sumudu transform and using the relation, if $R$ is a polynomial of degree less than $n$, then $S^{-1}[R(u) / P(u)]=R(\dot{D}) V$, then it follows that

$$
M_{P}(u) V[r-\varphi(y ; n)]=0
$$

for all $u$ sufficiently large (and hence for all real $u$ since the left side is a polynomial). From the relation $y \in \mathbb{C}^{n}$ and $M_{P}(x) y=0$ for all $x$, we have $y=0$. We conclude that $\gamma=\varphi(y ; n)$. This establishes existence.

The last term in the right-hand side of (2.20) can be written in the form of

$$
M_{P}(\dot{D}) V \gamma=\left(\begin{array}{llll}
V & V^{\prime} & \cdots & V^{(n-1)}
\end{array}\right)\left(\begin{array}{ccc}
a_{1} & a_{2} & \cdot \\
a_{2} & a_{3} & \cdot \\
a_{3} & \cdot & \cdot \\
\cdot & \cdot & \cdot \\
a_{n} & 0 & \cdot
\end{array}\right)\left(\begin{array}{ccc}
\cdot & \cdot & \cdot \\
\cdot & a_{n} & 0 \\
a_{n} & 0 & 0 \\
\cdot & \cdot & \cdot \\
\cdot & \cdot & 0
\end{array}\right)\left(\begin{array}{c}
\gamma_{0} \\
\gamma_{1} \\
\vdots \\
\gamma_{n-1}
\end{array}\right)
$$

for any vector $\gamma=\left(\gamma_{0}, \gamma_{1}, \ldots, \gamma_{n-1}\right) \in \mathbb{C}^{n}$. In the next section, we provide an example to make a comparison. 


\section{A Comparison on Solutions}

Consider that the steady-state temperature distribution function $f(x, y)$ in a long square bar with one face held at constant temperature $T_{0}$ and the other faces held at zero temperature is governed by the boundary-value problem

$$
\frac{\partial^{2} f(x, y)}{\partial x^{2}}+\frac{\partial^{2} f(x, y)}{\partial y^{2}}=0
$$

under the boundary conditions

$$
f(0, y)=0, \quad f(x, 0)=0, \quad f(x, \pi)=0, \quad f(\pi, y)=T_{0} .
$$

\subsection{Solution with Laplace Transform}

If we apply the multiple Laplace transform with respect to the variables $x$ and $y$ for (3.1), and single Laplace transform for the first and second boundary condition, we obtain

$$
\left(p^{2}+q^{2}\right) \overline{\bar{F}}(p, q)=\frac{\partial \bar{f}(0, q)}{\partial x}+\frac{\partial \bar{f}(p, 0)}{\partial y}
$$

If we look at (3.3) then we can easily notice that the right-hand side is transforms of the functions $\partial f(0, y) / \partial x$ and $\partial f(x, 0) / \partial y$ which are not among the boundary condition of (3.1). Hence, $\partial f(0, y) / \partial x$ and $\partial f(x, 0) / \partial y$ are taken to be the unknown functions $h(y)$ and $g(x)$, respectively. Then, by using single Laplace transform, we have

$$
\frac{\partial \bar{f}(0, q)}{\partial x}=H(q), \quad \frac{\partial \bar{f}(p, 0)}{\partial y}=G(p)
$$

By substituting (3.4) into (3.3) and rearrangement, we obtain

$$
\overline{\bar{F}}(p, q)=\frac{\mathrm{H}(q)}{\left(p^{2}+q^{2}\right)}+\frac{G(p)}{\left(p^{2}+q^{2}\right)} .
$$

On using single inverse Laplace transform with respect to $p$ for (3.3) and using the Laplace transform of convolution, we have

$$
\bar{f}(x, q)=H(q) \sin q x+\int_{0}^{x} G(\beta) \sin q(x-\beta) d \beta .
$$

Now, we take Laplace transform for third boundary condition and substitute in (3.6), using integral property

$$
\int_{0}^{\pi} Q(\beta) d \beta=\int_{0}^{x} Q(\beta) d \beta+\int_{x}^{\pi} Q(\beta) d \beta,
$$


and trigonometric manipulation, then (3.6) can be written in the form of

$$
\bar{f}(x, q)=-\frac{1}{\sin q \pi}\left[\sin q(x-\pi) \int_{0}^{x} G(\beta) \sin q \beta d \beta+\sin q x \int_{x}^{\pi} G(\beta) \sin q(\pi-\beta) d \beta\right] .
$$

In order to obtain the single inverse Laplace transform with respect to $q$, we use Cauchy's residue theorem, so we have a simple pole at $q= \pm n$; we use

$$
f(x, y)=\sum_{\text {residues }} e^{y q} \bar{f}(x, q) .
$$

We compute the residues at $q= \pm n$, and adding together, then we obtain

$$
f(x, y)=\sum_{\substack{n=1 \\ \text { odd }}}^{\infty} 2 \sin n x \sinh n y \int_{0}^{\pi} G(\beta) \sin n \beta d \beta,
$$

by using the last boundary condition and Fourier series to compute the unknown integral, then we obtain the solution of (3.1) as follows:

$$
f(x, y)=\left(\frac{4 T_{0}}{\pi}\right) \sum_{\substack{n=1 \\ \text { odd }}}^{\infty}\left(\frac{\sinh n y}{n \sinh n \pi}\right) \sin n x .
$$

\subsection{Solution with Sumudu Transform}

Now, we apply multiple Sumudu transform for the same problem to check the solution whether equal or not equal or probably does not exist. By applying multiple Sumudu transform for (3.1) as follows:

$$
\frac{1}{u^{2}} \overline{\bar{f}}(u, v)-\frac{1}{u^{2}} \bar{f}(0, v)-\frac{1}{u} \frac{\partial}{\partial x} \bar{f}(0, v)+\frac{1}{v^{2}} \overline{\bar{f}}(u, v)-\frac{1}{v^{2}} \bar{f}(u, 0)-\frac{1}{v} \frac{\bar{\partial}}{\partial y} f(u, 0)=0
$$

and by taking single Sumudu transform for first two boundary conditions of (3.2), we have

$$
\bar{f}(0, v)=0, \quad \bar{f}(u, 0)=0,
$$

where $f(x, 0)$ and $\partial f(0, y) / \partial x$ are taken to be unknown functions $h(y)$ and $g(x)$. Then, the Sumudu transform of unknown functions is given by

$$
\frac{\partial}{\partial x} \bar{f}(u, 0)=G(u), \quad \frac{\partial}{\partial x} \bar{f}(0, v)=H(v) .
$$


By substituting (3.13) and (3.14) into (3.12) and rearranging, we have

$$
\overline{\bar{f}}(u, v)=\frac{v u^{2} G(u)}{\left(u^{2}+v^{2}\right)}+\frac{u v^{2} H(v)}{\left(u^{2}+v^{2}\right)} .
$$

By taking inverse Sumudu transform with respect to $u$ for (3.15), and using convolution, we have

$$
\bar{f}(x, v)=\int_{0}^{x} G(\beta) \sin v(x-\beta) d \beta+v^{2} H(v) \sin v x .
$$

Now by taking single Sumudu transform for third boundary condition, we have $\bar{f}(u, \pi)=0$, and use $x=\pi$, we have

$$
H(v)=\frac{-\int_{0}^{\pi} G(\beta) \sin v(\pi-\beta) d \beta}{v^{2} \sin \pi v}
$$

Substituting (3.17) into (3.16), we obtain

$$
\bar{f}(x, v)=\int_{0}^{x} G(\beta) \sin v(x-\beta) d \beta+\sin v x\left[\frac{-\int_{0}^{\pi} G(\beta) \sin v(\pi-\beta) d \beta}{\sin \pi v}\right] .
$$

By using trigonometric properties, we have

$$
\bar{f}(x, v)=-\frac{1}{\sin \pi v}\left[\sin \pi v \int_{0}^{x} G(\beta) \sin v(x-\beta) d \beta+\sin v x \int_{0}^{\pi} G(\beta) \sin v(\pi-\beta) d \beta\right] .
$$

By rearrangement the above equation, we have

$$
\bar{f}(x, v)=-\frac{1}{\sin \pi v}\left[\sin v(x-\pi) \int_{0}^{x} G(\beta) \sin v \beta d \beta+\sin v x \int_{x}^{\pi} G(\beta) \sin v(\pi-\beta) d \beta\right] .
$$

In order to obtain inverse Sumudu transform for (3.20), we use the Cauchy's residue theorem, and then we have

$$
f(x, y)=\sum_{\text {residues }} e^{y q} \frac{\bar{f}(x, 1 / q)}{q} .
$$

If we replace the variable $v$ by $1 / q$ and divide the equation by $q$, then we obtain

$$
\bar{f}(x, v)=-\frac{1}{q \sin \pi / q}\left[\sin \frac{(x-\pi)}{q} \int_{0}^{x} G(\beta) \sin \frac{\beta}{q} d \beta+\sin \frac{x}{q} \int_{x}^{\pi} G(\beta) \sin \frac{(\pi-\beta)}{q} d \beta\right] .
$$


By using Cauchy's residue theorem, we have simple poles at $q=0$ and $q= \pm 1 / n$, thus it follows that the limit does not exist at $q=0$, then the solution does not exist. That leads us to make a remark that if the solution of differential equation by Laplace transform exists then it does not necessarily exist by using the Sumudu transform, but if the solution of differential equation by Sumudu transform exists then it necessarily exists by Laplace transform.

\section{Acknowledgment}

The authors gratefully acknowledge that this research was partially supported by University Putra Malaysia under the Research University Grant Scheme 05-01-09-0720RU.

\section{References}

[1] G. K. Watugala, "Sumudu transform: a new integral transform to solve differential equations and control engineering problems," International Journal of Mathematical Education in Science and Technology, vol. 24, no. 1, pp. 35-43, 1993.

[2] M. A. Aşiru, "Sumudu transform and the solution of integral equations of convolution type," International Journal of Mathematical Education in Science and Technology, vol. 32, no. 6, pp. 906-910, 2001.

[3] M. A. Aşiru, "Further properties of the Sumudu transform and its applications," International Journal of Mathematical Education in Science and Technology, vol. 33, no. 3, pp. 441-449, 2002.

[4] M. A. Aşiru, "Classroom note: application of the Sumudu transform to discrete dynamic systems," International Journal of Mathematical Education in Science and Technology, vol. 34, no. 6, pp. 944-949, 2003.

[5] F. B. M. Belgacem, A. A. Karaballi, and S. L. Kalla, "Analytical investigations of the Sumudu transform and applications to integral production equations," Mathematical Problems in Engineering, no. 3, pp. 103-118, 2003.

[6] H. Eltayeb, A. Kilıçman, and B. Fisher, "A new integral transform and associated distributions," Integral Transforms and Special Functions, vol. 21, no. 5-6, pp. 367-379, 2010.

[7] A. Kilıçman, V. G. Gupta, and B. Sharma, "On the solution of fractional Maxwell equations by Sumudu transform," Journal of Mathematics Research, vol. 2, no. 4, pp. 147-151, 2010.

[8] A. Kilıçman and H. Eltayeb, "A note on integral transforms and partial differential equations," Applied Mathematical Sciences, vol. 4, no. 3, pp. 109-118, 2010.

[9] A. Kadem, "Solving the one-dimensional neutron transport equation using Chebyshev polynomials and the Sumudu transform," Analele Universitatii din Oradea. Fascicola Matematica, vol. XII, pp. 153171, 2005.

[10] A. Kılıçman and H. Eltayeb, "On the applications of Laplace and Sumudu transforms," Journal of the Franklin Institute, vol. 347, no. 5, pp. 848-862, 2010.

[11] A. Kılıçman, H. Eltayeb, and R. P. Agarwal, "On Sumudu transform and system of differential equations," Abstract and Applied Analysis, vol. 2010, Article ID 598702, 11 pages, 2010.

[12] T. A. Estrin and T. J. Higgins, "The solution of boundary value problems by multiple Laplace transformations," Journal of the Franklin Institute, vol. 252, pp. 153-167, 1951.

[13] J. Zhang, "A Sumudu based algorithm for solving differential equations," Computer Science Journal of Moldova, vol. 15, no. 3, pp. 303-313, 2007.

[14] A. Kılıçman and H. E. Gadain, "An application of double Laplace transform and double Sumudu transform," Lobacherskii Journal of Mathematics, vol. 30, no. 3, pp. 214-223, 2009. 


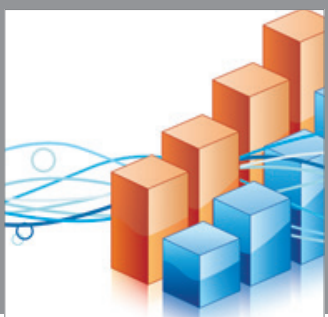

Advances in

Operations Research

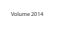

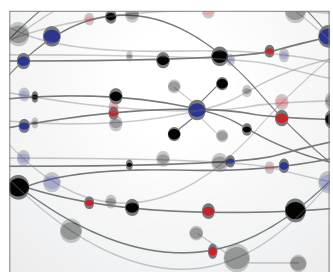

\section{The Scientific} World Journal
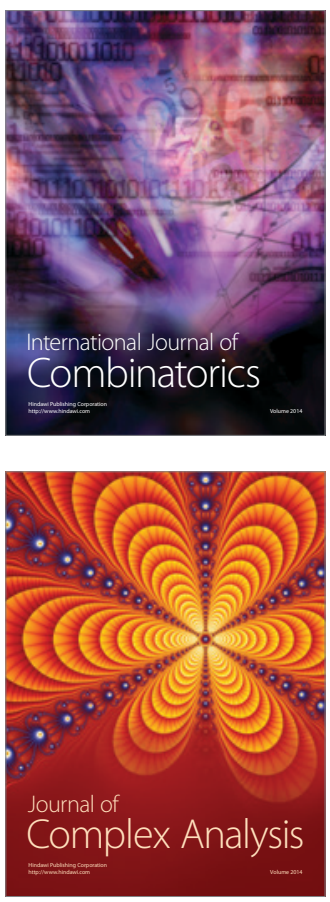

International Journal of

Mathematics and

Mathematical

Sciences
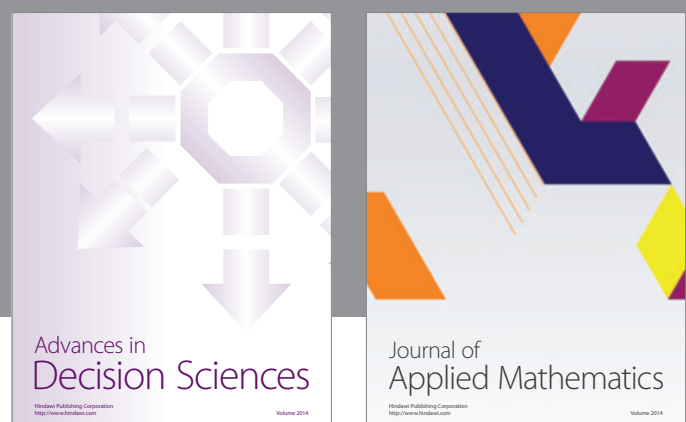

Journal of

Applied Mathematics
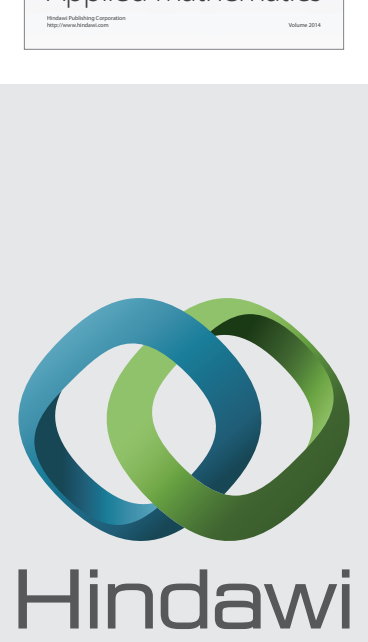

Submit your manuscripts at http://www.hindawi.com
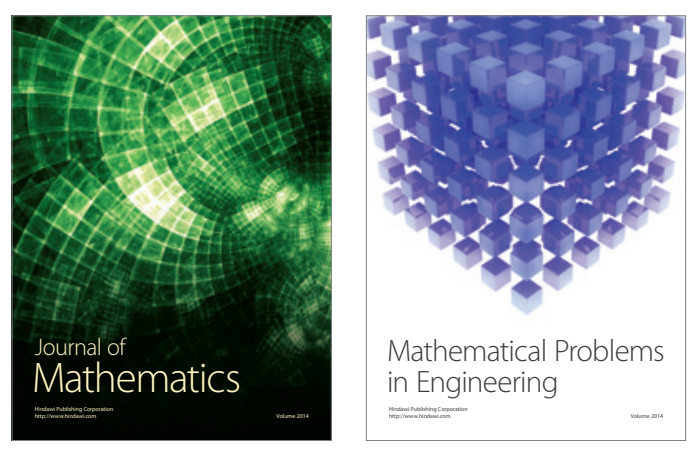

Mathematical Problems in Engineering
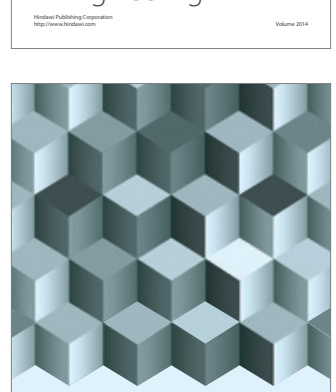

Journal of

Function Spaces
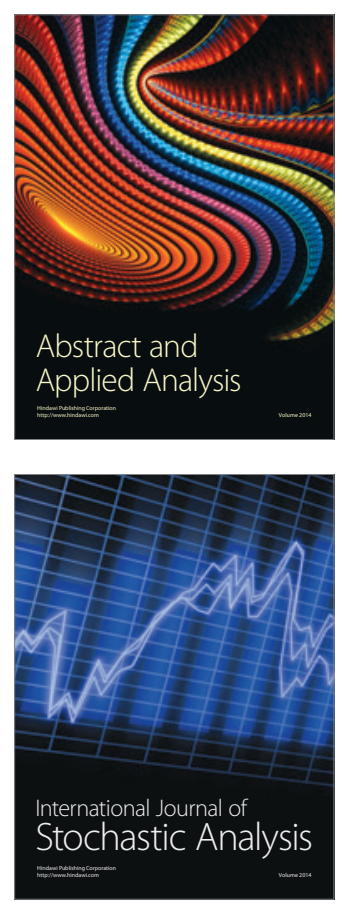

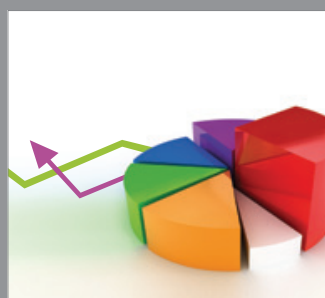

ournal of

Probability and Statistics

Promensencen
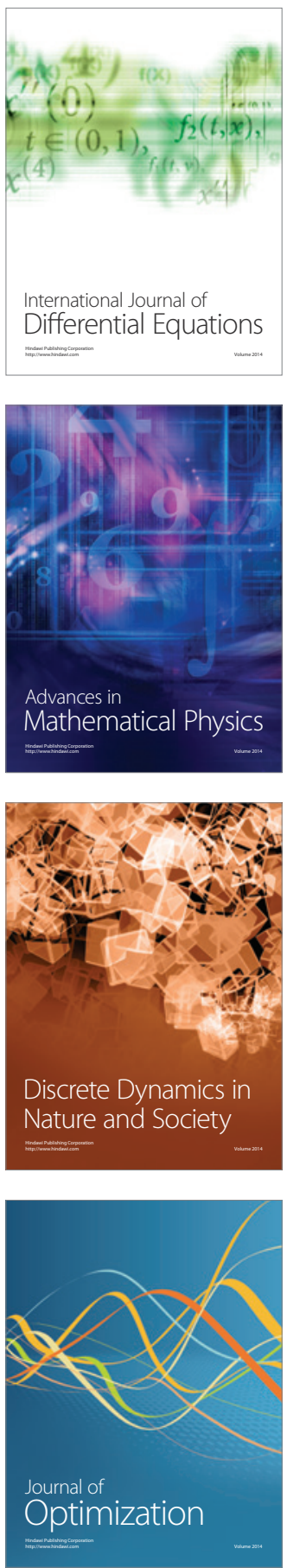